\title{
A novel hypothesis for the original functionality of the Visual Word Form Area: Processing shape sequences
}

\author{
*Carol Whitney, \\ Paddy Ross ${ }^{\mathrm{b}}$, \\ Zhiheng Zhou', \\ Lars Strother \\ ${ }^{a}$ Unaffiliated Researcher, Silver Spring, MD, USA \\ ${ }^{\mathrm{b}}$ Department of Psychology, Durham University, Durham, UK

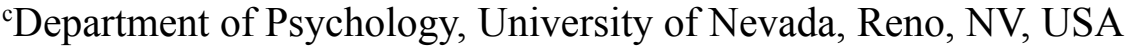 \\ *Correspondence: \\ Dr. Carol Whitney \\ cwhitney10@gmail.com
}




\section{Abstract}

There is ongoing debate about what characteristics of left ventral occipitotemporal cortex drive development of the Visual Word Form Area (VWFA). We offer a new hypothesis. A summary of occipitotemporal organization indicates that the VWFA falls in a cortical region supporting action analysis, rather than object recognition. We discuss evidence that letters are serially processed in a top-down manner during the initial years of reading acquisition, and propose that this sequential activation of letter representations causes the VWFA to develop in motionsensitive cortex specialized for processing of non-biological shape sequences. Supporting this hypothesis, a new fMRI analysis identifies a left-lateralized region that responds more strongly to dynamic motion of objects than humans; this region's location $(-48,-55,-8)$ falls almost exactly at the canonical VWFA coordinates $(-45,-57,-12)$.

Key Words: Visual Word Recognition; Orthographic; Reading Acquisition 


\section{Introduction}

The Visual Word Form Area (VWFA) is a region of left ventral Occipitotemporal Cortex (OTC) that is selective for letter strings (Dehaene \& Cohen, 2011), and is positioned at a similar cortical location across subjects (Vinckier et al., 2007). The question of what determines VWFA location is a topic of much interest, with many researchers assuming that the location is driven by pre-existing connectivity to language areas (Dehaene \& Dehaene-Lambertz, 2016; Hannagan, Amedi, Cohen, DehaeneLambertz, \& Dehaene, 2015; Pugh et al., 2013; Saygin et al., 2016; Schlaggar \& McCandliss, 2007; Stevens, Kravitz, Peng, Tessler, \& Martin, 2017; Yeatman, Rauschecker, \& Wandell, 2013).

However, the cortical region typically identified as the VWFA consists of two areas with different response profiles, namely the VWFA proper on the Occipitotemporal Sulcus/lateral Fusiform Gyrus, and the Lateral Inferotemporal Multimodal Area (LIMA) on the Inferior Temporal Gyrus. Both areas respond to visual words, but only the LIMA responds for phonological tasks on auditory words (Cohen, Jobert, Le Bihan, \& Dehaene, 2004; Danelli et al., 2017, 2013). The LIMA is thought to provide an interface between the visual and language systems (Cohen et al., 2004; Price \& Devlin, 2011). Correspondingly, the posterior Inferior Temporal Gyrus is associated with visual object naming (Herbet et al., 2016; Sebastian et al., 2014). Hence, the cortical location of the LIMA is indeed likely determined by connectivity to the language system.

But what determines the location of the VWFA? (Henceforth, 'VWFA' will refer to the VWFA proper.) We propose a novel answer to this question. First we review OTC organization, showing that the VWFA falls in a region selective for shape-based analysis of non-rigid motion. Then we outline acquisition of the skilled orthographic processing specified in the SERIOL2 model (Whitney, 2001, 2017; Whitney \& Marton, 2013), explaining the surprising location of the VWFA. In particular, we propose that serial letter processing causes the VWFA to develop in cortex specialized for recognizing temporal sequences of object shapes. We conclude with a discussion of alternative explanations of VWFA location, and avenues for future research.

\section{Organization of OTC}

In broad terms, ventral OTC supports object recognition (Culham et al., 2003; Goodale \& Milner, 1992) and lateral OTC supports action processing (Beauchamp, 2005; Lingnau \& Downing, 2015; 
Weiner \& Grill-Spector, 2013). At a finer scale, the OTC is organized around the properties of motion, shape, and animacy.

Figure 1 illustrates the selectivity of the OTC, and its legend defines abbreviations of cortical areas and structures used in the following text. The ventral region outlined in black responds more strongly to images of intact than scrambled objects (shape sensitivity) (Grill-Spector, 2003; Hasson, Harel, Levy, \& Malach, 2003; Jastorff \& Orban, 2009; Silson et al., 2013), in line with specialization for object processing. This area is known as the Lateral Occipital Complex (LOC). The lateral region outlined in blue responds more strongly to moving than still objects and humans (movement sensitivity), in line with the specialization for action perception and guidance (Beauchamp, Lee, Haxby, \& Martin, 2002, 2003; Costantini et al., 2005; Jastorff \& Orban, 2009).
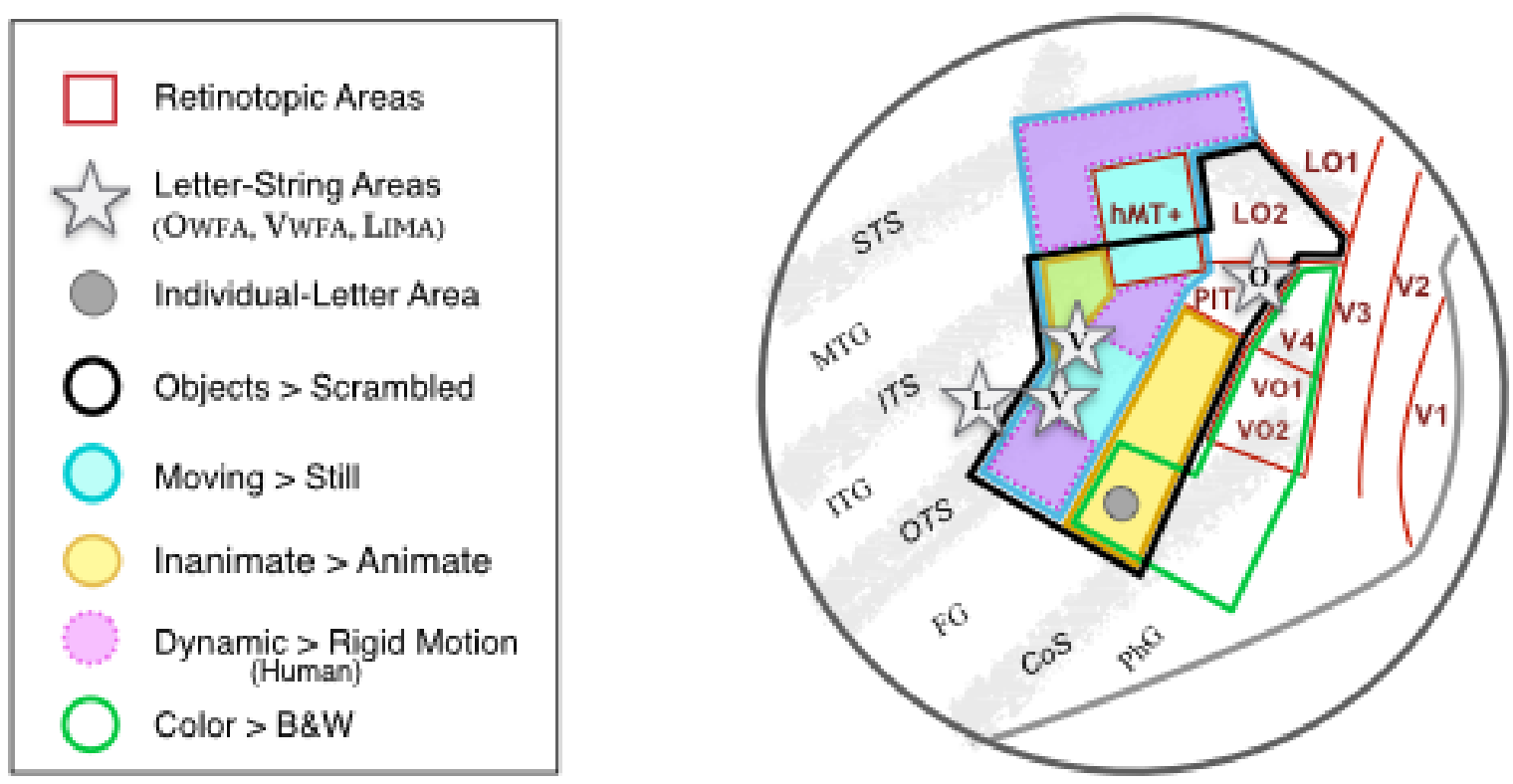

Figure 1: Idealized flattened cortex showing approximate locations of regions selective for motion, objects, animacy, and color. (For convenience, regions are drawn with straight lines.) Also shown are centers of letter-selective areas; the VWFA is broken into anterior and posterior functional parts, as discussed in the text. Locations of retinotopic areas relative to gyri are taken from Abdollahi et al. (2014). Locations of categorical regions based on references cited in the text. Abbreviations: STS Superior Temporal Sulcus; MTG Middle Temporal Gyrus; ITS Inferior Temporal Sulcus; ITG Inferior Temporal Gyrus; FG Fusiform Gyrus; CoS Collateral Sulcus; PhG Parahippocampal Gyrus; $h M T+$ human motion complex; LO1/2 Lateral Occipital areas 1/2; VO1/2 Ventral Occipital areas 1/2; PIT putative human homolog of macaque Posterior Inferotemporal area; OWFA Occipital Word Form Area; VWFA Visual Word Form Area; LIMA Lateral Inferotemporal Multimodal Area. 
Movement and shape sensitivity overlap in ventrolateral OTC. Hence, the OTC can be divided into three functional zones. The motion-sensitive dorsolateral OTC (DLOTC) lies on the STS and MTG. The motion- and shape-sensitive ventrolateral OTC (VLOTC) extends from the ITS to the lateral FG. The shape-sensitive ventromedial OTC (VMOTC) lies on the medial FG and CoS. VMOTC also houses the scene-selective Parahippocampal Place Area on the $\mathrm{CoS}$ and PhG (Epstein \& Kanwisher, 1998).

The border between the functionally-defined VLOTC and VMOTC runs along the center of the FG. Consistent with this split, the medial and lateral FG are comprised of separate cytoarchitectonic areas (Weiner et al., 2017). The split also aligns with patterns of resting-state functional connectivity. The lateral-FG/OTS is connected to temporal, parietal, and frontal regions involved in auditory analysis, motor control, and visual attention, consistent with specialization for action processing. The medial$\mathrm{FG} / \mathrm{CoS}$ is connected to anterior temporal regions involved in semantics and long-term memory, consistent with specialization for object and scene recognition (Hutchison, Culham, Everling, Flanagan, \& Gallivan, 2014).

In Figure 1, the gold region (running along medial FG) indicates a stronger response to inanimate objects than humans/animals, with no sensitivity to motion (Beauchamp et al., 2002; Konkle \& Caramazza, 2013; Stevens, Tessler, Peng, \& Martin, 2015; Wiggett, Pritchard, \& Downing, 2009). Sensitivity to color (color images $>$ B\&W images) is shown in green outline, which falls largely medial to the gold area (Lafer-Sousa, Conway, \& Kanwisher, 2016). Color is a property of the surfaces of objects. Other surface properties, such as texture and pattern, are also processed in this medial region (Cant \& Goodale, 2007; Cavina-Pratesi, Kentridge, Heywood, \& Milner, 2010). Color- and shapesensitivity overlap only in anterior medial shape-selective cortex (Lafer-Sousa et al., 2016); we refer to this overlap region as the amLOC. Hence, the amLOC likely integrates shape and surface properties for visual object recognition. Correspondingly, the amLOC region shows view-independent fMRI adaption for images of real objects, but not for drawings of made-up objects (Vuilleumier, Henson, Driver, \& Dolan, 2002).

The motion-sensitive region consists of areas with different preferences for animacy and type of motion. Animate entities move with changing shape (dynamic motion, such as a human walking), while inanimate objects often move with a fixed shape (rigid motion, such as a hammer in use). The greenish region (anterior to $\mathrm{hMT}+$ ) signifies selectivity for inanimate objects undergoing rigid motion. 
(Beauchamp et al., 2002, 2003). We refer to this as the Lateral Object Area (LOA). For static images, the LOA responds preferentially to small objects and tools (Bracci, Cavina-Pratesi, Connolly, \& Ietswaart, 2016; Bracci, Cavina-Pratesi, Ietswaart, Caramazza, \& Peelen, 2012; Hasson et al., 2003; Ishai, Ungerleider, Martin, Schouten, \& Haxby, 1999). This region also responds more strongly to images of hands than other body parts (Bracci et al., 2012). It is active for tactile shape recognition, unlike the amLOC (Amedi, Jacobson, Hendler, Malach, \& Zohary, 2002; Pietrini et al., 2004). These properties suggest that the LOA is specialized for interactions with objects.

The purplish areas signify regions that respond more strongly to dynamic human motion than to rigid motion of humans or tools (Beauchamp et al., 2002, 2003; Beauchamp \& Martin, 2007; Jastorff \& Orban, 2009). For static images, these areas respond more strongly to animals than inanimate objects (Konkle \& Caramazza, 2013; Wiggett et al., 2009). The purplish region above hMT + encompasses the dorsal portion of the Extrastriate Body Area (EBA) (Downing, Jiang, Shuman, \& Kanwisher, 2001; Weiner \& Grill-Spector, 2013), and the STS area selective for human motion (Allison, Puce, \& McCarthy, 2000). The region below hMT+ includes the Occipital Face Area and the ventral portion of the EBA (Rossion et al., 2003; Weiner \& Grill-Spector, 2010). The anterior purplish region corresponds to the Fusiform Face Area (Kanwisher, McDermott, \& Chun, 1997) and Fusiform Body Area (FBA) (Peelen \& Downing, 2005).

The FBA is identified more reliably in the right than left hemisphere (Peelen \& Downing, 2005), while a region that responds more strongly to hands than other body parts is observed in the left, but not right, hemisphere (Bracci, Ietswaart, Peelen, \& Cavina-Pratesi, 2010). The hand region overlaps the LOA (Bracci et al., 2012). Hence, left VLOTC may be specialized for integration of object and hand representations in action processing, resulting in less body-specific territory.

Dynamic motion can be neurally analyzed in two different ways. One is that motions of small edge segments are evaluated, and these local motion vectors are integrated. The other is that whole-object shape is evaluated at successive time points, like a sequence of snapshots, and the 'snapshots' are integrated. For example, point-light motion would preferentially drive motion-vector analysis, while slow presentation of an image sequence would preferentially drive shape-based analysis. In line with their selectivities, DLOTC primarily employs motion-vector analysis, and VLOTC shape-based analysis (Beauchamp et al., 2003; Jastorff \& Orban, 2009; Vangeneugden, Peelen, Tadin, \& Battelli, 2014). 
Regions in blue fill are motion-sensitive areas that do not show selectivity for human or rigid motion. The posterior blue area is $\mathrm{hMT}+$, the human retinotopic motion complex. (However, the EBA partially overlaps hMT+ (Ferri, Kolster, Jastorff, \& Orban, 2013; Ross, 2014), not shown here for clarity in delineating $\mathrm{hMT}+$.) In the macaque, MT+ is composed of four areas: MT, MST, V4t, and FST (Kolster et al., 2009). An fMRI study has shown that hMT+ has a similar organization (Kolster, Peeters, \& Orban, 2010). Putative human V4t and FST (phV4t/phFST) respond strongly to static objects, unlike human MT/MST (Kolster et al., 2010). The LOC overlaps ventral hMT+ (Kourtzi, Bülthoff, Erb, \& Grodd, 2002; Tootell, Echavarria, \& Nasr, 2015), corresponding to the location of phV4t/phFST. The shape selectivity of phV4t/phFST likely furnishes the rapid retinotopic 'snapshots' for shape-based motion analysis. Intracranially-recorded responses in VLOTC (and only in this cortical region) are sensitive to the order of two images presented with a Stimulus Onset Asynchrony of only $17 \mathrm{~ms}$ (Singer, Madsen, Anderson, \& Kreiman, 2015).

A second blue region is located on the OTS between the FBA and ventral EBA (approximate coordinates $-45,-55,-5$ in MNI space). Unlike the adjacent Body Areas, this region is not selective for full-body dynamic motion (Beauchamp \& Martin, 2007; Jastorff \& Orban, 2009). Unlike the nearby LOA, this region does not respond preferentially to rigid tool motion (Beauchamp \& Martin, 2007). So what is the sensitivity of this region of cortex? In a study that included dynamic tool motion, toolselective activation (versus dynamic human motion) was evident in this region, just ventral to the toolselective focus in the LOA (Safford, Hussey, Parasuraman, \& Thompson, 2010, Figure 2). This region responds more robustly to dynamic motion of invented 'Creatures' than of humans, and is the only such region to do so (Pyles, Garcia, Hoffman, \& Grossman, 2007). It responds more strongly to dynamic motion of hands or scissors than to static hands or scissors (Costantini et al., 2005). For static images, this region responds more robustly to small than large objects (Konkle \& Oliva, 2012). In the righthemisphere, this region is more responsive to animals than objects (Konkle \& Caramazza, 2013, Figure 4). In the left hemisphere, this region has no preference for animals versus objects (Konkle \& Caramazza, 2013, Figure 4), but is more more responsive to arms than other body parts (Orlov, Makin, \& Zohary, 2010, Figure 3).

Given this region's response profile and its location between areas involved in human full-body dynamic-motion processing, we suggest that it provides dynamic-motion analysis of other stimuli, such as forearms/hands, inanimate objects, and animals. The right hemisphere may be specialized for animal motion, whereas the left hemisphere may be specialized for objects and forearms, like the left LOA. We 
dub this left-hemisphere OTS region the Dynamic Object Area (DOA). The DOA may support recognition of actions with objects that undergo a shape change. For example, recognition of "opening a book" involves conjoint analysis of dynamic object and arm motion. This analysis would have to be sensitive to temporal order, to differentiate "opening" from "closing", for example. The location of the DOA in the VLOTC indicates that dynamic object motion is processed as a temporal sequence of shapes. Such processing would occur on a pathway from phV4t/phFST to the DOA.

Our account of the DOA indicates that it should respond more strongly to dynamic motion of real objects than of whole bodies. Such a comparison has not been published to our knowledge, but an existing fMRI data set is relevant. A study presented subjects with videos of dynamically-moving objects without people (e.g., metronomes, windmills), and of people performing meaningful motions (full bodies with faces blurred) (Ross, de Gelder, Crabbe, \& Grosbras, 2014). This study focused on cortical body areas, and the contrast (objects $>$ bodies) was not reported. Therefore, we undertook a reanalysis to perform this comparison. In 26 adult subjects, this contrast revealed large bilateral medial areas, corresponding to the inanimate-preferring VMOTC. We also found a 7-voxel cluster (FWE corrected $p<.05$ threshold with a peak- $t$ value of 4.87) at (-48 -55 -8) in MNI space, falling in the left OTS region. No object-preferring area was detected in right VLOTC.

This focal left OTS preference for objects falls in the DOA region, and does not extend into the more posterior LOA. Recall that the LOA shows an inanimate-object preference when stimuli are static or undergoing rigid motion (Beauchamp \& Martin, 2007; Konkle \& Caramazza, 2013). Hence, the LOA's selectivity for inanimate objects appears specific to stimuli not undergoing dynamic motion. Recall also that the DOA does not show an inanimate-object preference when stimuli are static or undergoing rigid motion (Beauchamp \& Martin, 2007; Konkle \& Caramazza, 2013). Hence, this region's selectivity for inanimate objects appears specific to stimuli that are undergoing dynamic motion. These response patterns indicate that this left OTS region indeed houses a Dynamic Object Area.

Next we consider selectivity to visual words. In Figure 1, stars illustrate the centers of regions involved in orthographic processing. The VWFA can be divided into anterior (aVWFA) and posterior (pVWFA) parts, based on response profiles. The pVWFA is sensitive to low-level motion (random movement of a low-spatial-frequency Gabor patch), while the aVWFA is not (Danelli et al., 2017, 2013). The pVWFA 
houses sublexical orthographic representations (Dehaene et al., 2004), while the aVWFA encodes whole words (Glezer, Jiang, \& Riesenhuber, 2009; Glezer, Kim, Rule, Jiang, \& Riesenhuber, 2015).

A posterior region on the Inferior Occipital Gyrus also participates in visual word recognition, dubbed the Occipital Word Form Area (OWFA) (Strother, Coros, \& Vilis, 2016). This left-hemisphere area encodes letters that fall in foveal/parafoveal Left Visual Field, but does not encode animal shapes falling in this same retinal region (Strother, Zhou, Coros, \& Vilis, 2017). Hence, this area is tuned for orthographic processing. The OWFA is located between V4 and hMT+, corresponding to a shapeselective retinotopic region (phPIT) that is the putative human analog of the macaque Posterior Inferotemporal area (Kolster et al., 2010).

A priori, one would expect visual words to be identified in an area specialized for object recognition, namely the amLOC. Indeed, recognition of individual letters activates the amLOC region (James, James, Jobard, Wong, \& Gauthier, 2005). However, the aVWFA is not located in the amLOC, but rather falls in the OTS/lateral-FG (Cohen et al., 2004; Danelli et al., 2017, 2013), in the DOA region.

However, a recent intracranial study also detected visual-word responses in the medial FG/CoS (Lochy et al., 2018). The words used in this study were high-frequency nouns. We suggest that these words activated representations of their corresponding objects in the amLOC. Indeed, activity in the medial FG is reduced for passive reading of noun pairs from the same semantic category (e.g., pig, fox) versus different categories (e.g., desk, lion), likely indicating that visual words implicitly activate object imagery in medial FG (Wheatley, Weisberg, Beauchamp, \& Martin, 2005).

In an fMRI study that examined the instantiation of new Visual Word Forms by training subjects on a set of pseudowords, effects for trained versus untrained pseudowords were found only in the lateralFG/OTS/ITG (Glezer et al., 2015), indicating that the medial FG does not house Visual Word Forms. Furthermore, across reading development, distributed fMRI responses to letter strings become more informative and distinctive within VLOTC, but not within VMOTC (Nordt et al., 2018), again indicating the Visual Word Forms do not develop in the medial FG.

Why would a visual word, which is a static stimulus, be identified in a region associated with analysis of dynamic movement? The SERIOL2 model of orthographic processing (Whitney \& Marton, 2013) proposes that Letters are activated serially during visual word recognition. ('Letter' with a capital 'L' denotes the neural representation of a letter.) That is, a visual word is processed as a temporal sequence of object shapes (Letters). Therefore, the VWFA falls in cortex that processes shape sequences. 


\section{Reading acquisition}

Next we consider development of the Visual Word Form system in more detail. Initially, children learn to recognize letters. They also interact with letters by writing them, associating them with speech sounds, and manipulating physical letters. Therefore, Letter representations develop in the amLOC for letter recognition and in the LOA for interactions with letters.

Then children are taught to read words. Based on fixation patterns and word-length effects on reading Reaction Times for short words ( 2 to 5 letters), the acquisition of skilled orthographic processing can be divided into three phases. In the first phase, the length effect is $\sim 200 \mathrm{~ms} /$ letter and saccade distance is one letter, indicating that the child fixates every letter of a word (De Luca, Zeri, Spinelli, \& Zoccolotti, 2010; Marinus \& de Jong, 2010; Zoccolotti et al., 2005).

Initially, words are sounded out via conscious knowledge of grapheme-phoneme correspondences. Phonemes are represented in the STS (Liebenthal, Binder, Spitzer, Possing, \& Medler, 2005). U-fiber connections between the posterior ITG/ITS and MTG/STS (Oishi et al., 2011) support audiovisual integration of object movements and sounds, such as for tools (Beauchamp, Lee, Argall, \& Martin, 2004). This connectivity would allow development of direct associations between Letters in the LOA and Phonemes in the STS. Once such connections are established, Letter $\rightarrow$ Phoneme activation is automatic and fast (Blomert, 2011). Therefore, the rate of letter processing can be increased.

Indeed, in the second orthographic phase (which starts at the end of first grade and lasts until third grade), the length effect is $\sim 50 \mathrm{~ms} /$ letter and saccade distance is about five letters (De Luca et al., 2010; Marinus \& de Jong, 2010; Zoccolotti et al., 2005). The magnitude of the length effect suggests that children covertly shift visual attention (Buschman \& Miller, 2009) across letters within a fixation. Correspondingly, early reading expertise is positively associated with activity in attention-related brain areas (Boros et al., 2016; Pugh et al., 2013).

The initial ventral occipitotemporal specialization for reading would develop at the Letter level in the LOA, via development of connections to Phonemes (Brem et al., 2010). During the first and second orthographic phases, letter processing is obviously serial. We propose that the pathway for processing dynamic object motion is recruited to encode the sequences of Letters activated in the LOA. That is, Visual Word Forms (VWFs), which recognize Letter sequences, develop in the DOA. We assume that posterior DOA encodes short sequences of shapes, which are integrated and recognized in anterior DOA. In the case of Letters, the representation in the posterior DOA would correspond to the Open- 
Bigrams of the SERIOL2 model (Whitney \& Marton, 2013), which encode ordered letter pairs. Hence, we propose that the sublexical pVWFA develops in the LOA and posterior DOA, while the lexical aVWFA develops in the anterior DOA.

A longitudinal fMRI study has been performed in which children's cortical selectivity to written words and other visual categories was analyzed prior to reading instruction, and then throughout the first grade to the second grade (Dehaene-Lambertz, Monzalvo, \& Dehaene, 2018). Pre-reading, the children showed a lateral region with a preferential response to tool images, corresponding to the LOA. The children also showed medial tool-responsive region, corresponding to the amLOC (Dehaene-Lambertz et al., 2018, Figure 2). In line with our account, the initial ventral occipitotemporal specialization for reading developed within the lateral tool region. At this location, responses to visual words increased through most of first grade, but decreased at the end of first grade into the second grade. Visual-word responses in the posterior STS yielded the same response pattern. The period of decreasing activity suggests increased efficiency of Letter and Phoneme activation. Indeed, its timing aligns exactly with the onset of the second orthographic phase. In contrast, at the canonical VWFA location, visual-word responses monotonically increased from first to second grade, indicating ongoing instantiation of VWFs.

The third orthographic phase (which starts around fourth grade) is signaled by the absence of a length effect (De Luca et al., 2010; Gagl, Hawelka, \& Wimmer, 2015; Marinus \& de Jong, 2010; Zoccolotti et al., 2005). We propose that the top-down attention shifts (in the second phase) drive visual perceptual learning that allows automatic serial Letter activation (without attention shifts) in the third phase. This learning is specific to the retinal locations evaluated within a fixation during the second phase. Indeed, at onset of the third phase, saccade distance remains the same as in the second phase (Gagl et al., 2015), consistent with a shift to automatic serialization learned only for the retinal locations processed in the second phase. Hence, we propose that specialized orthographic processing develops in anterior retinotopic shape-selective cortex. That is, the OWFA forms in phPIT. In the third phase, successive Letters are taken to be activated on successive gamma cycles, at a rate of around $16 \mathrm{~ms} /$ letter (Whitney \& Marton, 2013). As indicated above, VLOTC is sensitive to order at this time scale (Singer et al., 2015).

The serial Letter activation provides the input to the lexical pathway (into the aVWFA) and to the sublexical pathway (into the STS). As Whitney (2017) explains, rapid serial Letter activation does not 
necessarily cause a length effect on the lexical pathway because Letters are jointly mapped to a single outcome (the VWF), and reaction times are dominated by settling dynamics in the VWF network. Hence, absence of a length effect for word recognition does not necessarily imply parallel Letter processing. In contrast, on the sublexical pathway, serial Letter activation necessarily causes a length effect (as observed for pseudoword naming) because Letters are individually mapped to Phonemes. However, due to the absence of a lexical length effect in skilled readers, most researchers assume that VWFs are activated by a parallel Letter encoding (Whitney, 2017). This conclusion is inconsistent with reading acquisition. We have seen that VWFs develop when letters are obviously serially processed. Serial activation of Letters provides an abstract (non-retinotopic) representation of letter order. VWFs would develop based on this encoding. To maintain continuity of neural encoding, skilled reading must utilize the same serial representation of letter order.

Note that the claim is not that letters are recognized serially. In SERIOL2, letter recognition occurs in parallel in retinotopic cortex (now taken to be in phPIT). Automatic serialization operates spatially across these retinotopic Letter representations to sequentially activate abstract Letter representations (in pVWFA). Hence, the claim is actually that a serial Letter encoding provides the input to VWFs.

The acquired retinotopic processing that yields automatic serialization is specific to the typical horizontal format. If a word is presented to a skilled reader in an unusual format (e.g., vertically or horizontally with two spaces between letters), automatic serialization fails. Instead, letters are selected with sequential attention shifts (Cohen, Dehaene, Vinckier, Jobert, \& Montavont, 2008), as in the second orthographic phase. The ability of the adult reading system to handle such input indicates that the VWFA 'expects' a serial encoding of letter order.

In summary, we propose that the VLOTC pathway for recognition of shape sequences is recruited for visual word recognition. Letter representations in the LOA support direct Letter-Phoneme mapping, becoming part of the pVWFA. Sequences of Letters are recognized in the anterior DOA, becoming the aVWFA. In the second orthographic phase, retinotopic processing specific to letter strings is acquired, and the OWFA develops in phPIT. In the amLOC, letters are encoded as individual objects (James et al., 2005).

In an intracranial study, evidence was found for Letter representations stretching from the Inferior Occipital Gyrus to the amLOC region (Lochy et al., 2018). Due to the presence of anterior Letter representations, the authors concluded that visual word recognition is not hierarchical. However, we 
suggest that the anterior Letter representations encode Letters as individual objects, and such Letter representations do not participate in visual word recognition. Rather, word recognition is indeed hierarchical, from retinotopic Letters in the OWFA, to abstract Letters and Open-Bigrams in the pVWFA, to VWFs in the aVWFA.

\section{Discussion}

We propose that serial letter processing during reading acquisition causes the VWFA to develop in cortex that encodes temporal sequences of shapes. Therefore, the VWFA 'expects' a serial encoding of letter position, which is maintained in skilled reading via a learned mechanism that automatically serially activates abstract Letter representations, as specified in the SERIOL2 model (Whitney \& Marton, 2013). Our account provides a natural explanation of why the VWFA is located in cortex selective for moving objects.

\subsection{Alternative accounts}

Vogel, Petersen and Schlaggar (2014) have suggested that the VWFA falls in a cortical region that processes grouped objects via attentional selection. Similarly, we propose that the location of the VWFA is related to serial attentional selection of letters within a string. However, based on our review of OTC selectivity, we believe that this region's original function is to recognize shape sequences generated by non-rigid motion. We attribute this region's connectivity to the dorsal attention system (Vogel et al., 2014) as supporting visual selection during action processing.

The results of a longitudinal study (Saygin et al., 2016) have been taken as showing that VWFA location is determined by pre-existing connectivity to the language system. In this study, brain imaging was performed in five-year olds, and then VWFA, Face, and Object ROIs were defined at eight years. The white-matter connectivities of these ROIs were then examined in the scans taken at five years. The authors found that the VWFA ROI had stronger connectivity to language regions than did the nearby Face and Object ROIs. However, this study did not differentiate between the VWFA and the LIMA. The VWFA ROI was defined as (visual words $>$ objects). Therefore, it would tend to omit voxels that also respond strongly to objects. Indeed, their VWFA ROI showed no response to objects or faces. In contrast, in a study where the VWFA ROI was defined as (visual words $>$ scrambled words), the ROI showed a robust response to objects and faces (with an even stronger response to visual words) (Glezer \& Riesenhuber, 2013). In the Saygin et al. study, word response was not any stronger in the VWFA 
ROI than the Object or Face ROIs. These results suggest that the Saygin et al. VWFA criterion neglected word-responsive voxels within visual cortex (i.e., in the VWFA proper), and primarily identified voxels in the LIMA. As an interface region, it is only to be expected that the LIMA would show different white-matter connectivity than the Object/Face ROIs in visual cortex. Hence, this study likely does not address what determines VWFA location in visual cortex.

Studies of selectivity for object size have found that the VLOTC has a foveal bias and prefers small objects, and the VMOTC has a peripheral bias and prefers large objects (Hasson et al., 2003; Konkle \& Caramazza, 2013; Konkle \& Oliva, 2012). Could these biases explain the VLOTC location of the VWFA, as suggested by Nordt et al. (2018)? The VMOTC also responds strongly to small objects, such as tools and individual letters (Beauchamp \& Martin, 2007; Creem-Regehr \& Lee, 2005; James et al., 2005). The response to large objects overlaps with selectivity to scenes (He et al., 2013). These results suggest that the Parahippocampal Place Area responds preferentially to large objects, while the amLOC responds to objects of all sizes. Hence, size cannot explain why the VWFA does not form in the amLOC.

We have suggested that the VWFA falls in a cortical area that analyzes arm/hand motions (for interactions with objects). Similarly, Dehaene and Dehaene-Lambertz (2016) have suggested that the VWFA falls in a region specialized for processing co-speech gestures, attributing this localization to presumed access to the language system. However, the VMOTC object-recognition system has access to the language system via the posterior ITG, which has been implicated in object naming (Herbet et al., 2016; Sebastian et al., 2014). A priori, visual word recognition seems more similar to object recognition than gesture analysis. Why then wouldn't the VWFA develop in VMOTC and access the object-naming pathway? We believe that our account based on sequential processing provides a compelling explanation of why the VWFA falls in VLOTC rather than VMOTC.

\subsection{Future research}

Our analysis implies that that the DOA should respond more strongly to dynamic than rigid motion of objects. A study to directly evaluate this comparison should be performed. The contrast (dynamic $>$ rigid) should isolate the DOA, while (rigid $>$ dynamic) should isolate the LOA. Neither contrast should yield VMOTC activation. 
Additional research is required to clarify the selectivity of cortical region of the DOA. Are parts of this area differently responsive to dynamic motion of forearms, small objects, or big objects? Is the righthemisphere homolog indeed selective for animal motion? If so, are different parts of the righthemisphere area selective for different modes of locomotion, such as walking versus flying?

In skilled readers, the VWFA and the DOA should overlap within subjects. To evaluate the feasibility of isolating the DOA in individual subjects, further analysis of the Ross et al. (2014) data was carried out. We performed a region of interest (ROI) analysis surrounding the average coordinate of the peak voxel (for objects $>$ bodies in VLOTC) across all subjects. This allowed us to run a peak- $t$ value extraction algorithm at various statistical thresholds to determine the size and specificity of this area in individual subjects. We found that, at an uncorrected $p<.001$ threshold, the area was identifiable in 14/26 adults, with an average extent of 16 voxels. Figure 2 illustrates, on partially-inflated cortex, the location of the peak voxel from each subject, and the approximate locations of the OWFA, pVWFA, aVWFA, LIMA, $\mathrm{hMT}+$, and LOA. The individual peaks largely fall within the pVWFA region.

This gives compelling evidence that, even using experimental data not designed to isolate the DOA, it can be reliably identified in half of adult subjects. Future research will identify the best way to delineate the DOA, and locations of the VWFA and DOA will be compared within subjects.

A broad area for future research is to investigate the implications of our account for impaired reading acquisition. For example, if sequential attentional selection of individual letters is indeed necessary for normal VWFA development, inability to focus and shift attention from letter to letter could preclude VWFA formation and lead to developmental dyslexia. 


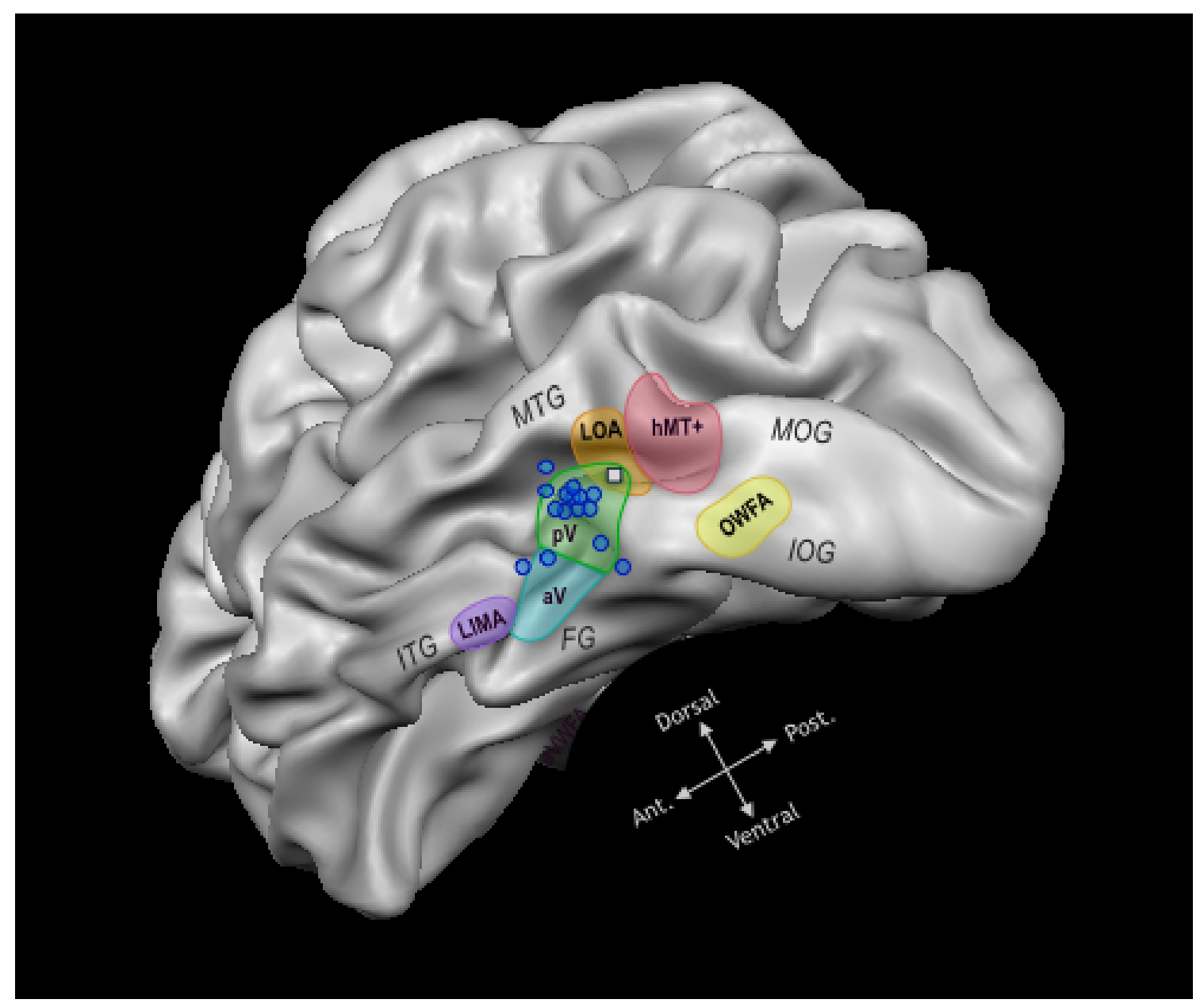

Figure 2: Blue dots show locations of individual VLOTC peaks for (objects $>$ bodies) from reanalysis of Ross et al. (2014) data. The small white square with black outline shows the location of the initial VLOTC specialization for reading (Dehaene-Lambertz et al., 2018). Locations of hMT+ and functional areas derived from corresponding references in the text. New abbreviations: MOG Middle Occipital Gyrus; IOG Inferior Occipital Gyrus; $p V$ pVWFA; $a V$ aVWFA.

\section{Conclusion}

We propose that serial processing of letters in the early years of reading acquisition causes the VWFA to develop in a VLOTC region specialized for recognition of shape sequences. Specifying how and why the VWFA typically develops in VLOTC is an important issue in understanding normal and impaired reading acquisition. We hope that our novel account of VWFA formation will contribute to such understanding. 
Acknowledgments: We thank Marc Brysbaert for helpful comments.

Funding: This research did not receive any specific grant from funding agencies in the public, commercial, or not-for-profit sectors.

Declarations of interest: none 


\section{References}

Abdollahi, R. O., Kolster, H., Glasser, M. F., Robinson, E. C., Coalson, T. S., Dierker, D., ... Orban, G. A. (2014). Correspondences between retinotopic areas and myelin maps in human visual cortex. NeuroImage, 99, 509-524. https://doi.org/10.1016/j.neuroimage.2014.06.042

Allison, T., Puce, A., \& McCarthy, G. (2000). Social perception from visual cues: role of the STS region. Trends in Cognitive Sciences, 4(7), 267-278. https://doi.org/10.1016/S13646613(00)01501-1

Amedi, A., Jacobson, G., Hendler, T., Malach, R., \& Zohary, E. (2002). Convergence of Visual and Tactile Shape Processing in the Human Lateral Occipital Complex. Cerebral Cortex, 12(11), 1202-1212. https://doi.org/10.1093/cercor/12.11.1202

Beauchamp, M. S. (2005). See me, hear me, touch me: multisensory integration in lateral occipitaltemporal cortex. Current Opinion in Neurobiology, 15(2), 145-153.

https://doi.org/10.1016/j.conb.2005.03.011

Beauchamp, M. S., Lee, K. E., Argall, B. D., \& Martin, A. (2004). Integration of Auditory and Visual Information about Objects in Superior Temporal Sulcus. Neuron, 41(5), 809-823. https://doi.org/10.1016/S0896-6273(04)00070-4

Beauchamp, M. S., Lee, K. E., Haxby, J. V., \& Martin, A. (2002). Parallel Visual Motion Processing Streams for Manipulable Objects and Human Movements. Neuron, 34(1), 149-159. https://doi.org/10.1016/S0896-6273(02)00642-6

Beauchamp, M. S., Lee, K. E., Haxby, J. V., \& Martin, A. (2003). FMRI responses to video and pointlight displays of moving humans and manipulable objects. Journal of Cognitive Neuroscience, 15(7), 991-1001. https://doi.org/10.1162/089892903770007380

Beauchamp, M. S., \& Martin, A. (2007). Grounding Object Concepts in Perception and Action: Evidence from FMRI Studies of Tools. Cortex, 43(3), 461-468. https://doi.org/10.1016/S00109452(08)70470-2

Blomert, L. (2011). The neural signature of orthographic-phonological binding in successful and failing reading development. NeuroImage, 57(3), 695-703.

https://doi.org/10.1016/j.neuroimage.2010.11.003

Boros, M., Anton, J.-L., Pech-Georgel, C., Grainger, J., Szwed, M., \& Ziegler, J. C. (2016). Orthographic processing deficits in developmental dyslexia: Beyond the ventral visual stream. NeuroImage, 128, 316-327. https://doi.org/10.1016/j.neuroimage.2016.01.014

Bracci, S., Cavina-Pratesi, C., Connolly, J. D., \& Ietswaart, M. (2016). Representational content of occipitotemporal and parietal tool areas. Neuropsychologia, 84, 81-88.

https://doi.org/10.1016/j.neuropsychologia.2015.09.001

Bracci, S., Cavina-Pratesi, C., Ietswaart, M., Caramazza, A., \& Peelen, M. V. (2012). Closely overlapping responses to tools and hands in left lateral occipitotemporal cortex. Journal of Neurophysiology, 107(5), 1443-1456. https://doi.org/10.1152/jn.00619.2011 
Bracci, S., Ietswaart, M., Peelen, M. V., \& Cavina-Pratesi, C. (2010). Dissociable Neural Responses to Hands and Non-Hand Body Parts in Human Left Extrastriate Visual Cortex. Journal of Neurophysiology, 103(6), 3389-3397. https://doi.org/10.1152/jn.00215.2010

Brem, S., Bach, S., Kucian, K., Guttorm, T. K., Martin, E., Lyytinen, H., ... Richardson, U. (2010). Brain sensitivity to print emerges when children learn letter-speech sound correspondences. Proceedings of the National Academy of Sciences of the United States of America, 107(17), 7939-7944. https://doi.org/10.1073/pnas.0904402107

Buschman, T. J., \& Miller, E. K. (2009). Serial, Covert Shifts of Attention during Visual Search Are Reflected by the Frontal Eye Fields and Correlated with Population Oscillations. Neuron, 63(3), 386-396. https://doi.org/10.1016/j.neuron.2009.06.020

Cant, J. S., \& Goodale, M. A. (2007). Attention to form or surface properties modulates different regions of human occipitotemporal cortex. Cerebral Cortex (New York, N.Y.: 1991), 17(3), 713731. https://doi.org/10.1093/cercor/bhk022

Cavina-Pratesi, C., Kentridge, R. W., Heywood, C. A., \& Milner, A. D. (2010). Separate Channels for Processing Form, Texture, and Color: Evidence from fMRI Adaptation and Visual Object Agnosia. Cerebral Cortex, 20(10), 2319-2332. https://doi.org/10.1093/cercor/bhp298

Cohen, L., Dehaene, S., Vinckier, F., Jobert, A., \& Montavont, A. (2008). Reading normal and degraded words: contribution of the dorsal and ventral visual pathways. NeuroImage, 40(1), 353-366. https://doi.org/10.1016/j.neuroimage.2007.11.036

Cohen, L., Jobert, A., Le Bihan, D., \& Dehaene, S. (2004). Distinct unimodal and multimodal regions for word processing in the left temporal cortex. NeuroImage, 23(4), 1256-1270. https://doi.org/ 10.1016/j.neuroimage.2004.07.052

Costantini, M., Galati, G., Ferretti, A., Caulo, M., Tartaro, A., Romani, G. L., \& Aglioti, S. M. (2005). Neural Systems Underlying Observation of Humanly Impossible Movements: An fMRI Study. Cerebral Cortex, 15(11), 1761-1767. https://doi.org/10.1093/cercor/bhi053

Creem-Regehr, S. H., \& Lee, J. N. (2005). Neural representations of graspable objects: are tools special? Cognitive Brain Research, 22(3), 457-469. https://doi.org/10.1016/j.cogbrainres.2004.10.006

Culham, J. C., Danckert, S. L., Souza, J. F. X. D., Gati, J. S., Menon, R. S., \& Goodale, M. A. (2003). Visually guided grasping produces fMRI activation in dorsal but not ventral stream brain areas. Experimental Brain Research, 153(2), 180-189. https://doi.org/10.1007/s00221-003-1591-5

Danelli, L., Berlingeri, M., Bottini, G., Borghese, N. A., Lucchese, M., Sberna, M., ... Paulesu, E. (2017). How many deficits in the same dyslexic brains? A behavioural and fMRI assessment of comorbidity in adult dyslexics. Cortex, 97, 125-142. https://doi.org/10.1016/j.cortex.2017.08.038

Danelli, L., Berlingeri, M., Bottini, G., Ferri, F., Vacchi, L., Sberna, M., \& Paulesu, E. (2013). Neural intersections of the phonological, visual magnocellular and motor/cerebellar systems in normal readers: Implications for imaging studies on dyslexia. Human Brain Mapping, 34(10), 26692687. https://doi.org/10.1002/hbm.22098

De Luca, M., Zeri, F., Spinelli, D., \& Zoccolotti, P. (2010). The acquisition of reading fluency in an orthographically transparent language (Italian): an eye movement longitudinal study. Medical 
Science Monitor: International Medical Journal of Experimental and Clinical Research, 16(3), SC1-7.

Dehaene, S., \& Cohen, L. (2011). The unique role of the visual word form area in reading. Trends in Cognitive Sciences, 15(6), 254-262. https://doi.org/10.1016/j.tics.2011.04.003

Dehaene, S., \& Dehaene-Lambertz, G. (2016). Is the brain prewired for letters? Nature Neuroscience, 19(9), 1192-1193. https://doi.org/10.1038/nn.4369

Dehaene, S., Jobert, A., Naccache, L., Ciuciu, P., Poline, J.-B., Le Bihan, D., \& Cohen, L. (2004). Letter binding and invariant recognition of masked words: behavioral and neuroimaging evidence. Psychological Science: A Journal of the American Psychological Society / APS, 15(5), 307-313. https://doi.org/10.1111/j.0956-7976.2004.00674.x

Dehaene-Lambertz, G., Monzalvo, K., \& Dehaene, S. (2018). The emergence of the visual word form: Longitudinal evolution of category-specific ventral visual areas during reading acquisition. PLOS Biology, 16(3), e2004103. https://doi.org/10.1371/journal.pbio.2004103

Downing, P. E., Jiang, Y., Shuman, M., \& Kanwisher, N. (2001). A Cortical Area Selective for Visual Processing of the Human Body. Science, 293(5539), 2470-2473. https://doi.org/10.1126/science.1063414

Epstein, R., \& Kanwisher, N. (1998). A cortical representation of the local visual environment. Nature, 392(6676), 598-601. https://doi.org/10.1038/33402

Ferri, S., Kolster, H., Jastorff, J., \& Orban, G. A. (2013). The overlap of the EBA and the MT/V5 cluster. NeuroImage, 66, 412-425. https://doi.org/10.1016/j.neuroimage.2012.10.060

Gagl, B., Hawelka, S., \& Wimmer, H. (2015). On Sources of the Word Length Effect in Young Readers. Scientific Studies of Reading, 19(4), 289-306. https://doi.org/10.1080/10888438.2015.1026969

Glezer, L. S., Jiang, X., \& Riesenhuber, M. (2009). Evidence for highly selective neuronal tuning to whole words in the "visual word form area." Neuron, 62(2), 199-204. https://doi.org/10.1016/j.neuron.2009.03.017

Glezer, L. S., Kim, J., Rule, J., Jiang, X., \& Riesenhuber, M. (2015). Adding Words to the Brain’s Visual Dictionary: Novel Word Learning Selectively Sharpens Orthographic Representations in the VWFA. Journal of Neuroscience, 35(12), 4965-4972.

https://doi.org/10.1523/JNEUROSCI.4031-14.2015

Glezer, L. S., \& Riesenhuber, M. (2013). Individual Variability in Location Impacts Orthographic Selectivity in the "Visual Word Form Area." Journal of Neuroscience, 33(27), 11221-11226. https://doi.org/10.1523/JNEUROSCI.5002-12.2013

Goodale, M. A., \& Milner, A. D. (1992). Separate visual pathways for perception and action. Trends in Neurosciences, 15(1), 20-25. https://doi.org/10.1016/0166-2236(92)90344-8

Grill-Spector, K. (2003). The neural basis of object perception. Current Opinion in Neurobiology, 13(2), 159-166. https://doi.org/10.1016/S0959-4388(03)00040-0

Hannagan, T., Amedi, A., Cohen, L., Dehaene-Lambertz, G., \& Dehaene, S. (2015). Origins of the specialization for letters and numbers in ventral occipitotemporal cortex. Trends in Cognitive Sciences, 19(7), 374-382. https://doi.org/10.1016/j.tics.2015.05.006 
Hasson, U., Harel, M., Levy, I., \& Malach, R. (2003). Large-Scale Mirror-Symmetry Organization of Human Occipito-Temporal Object Areas. Neuron, 37(6), 1027-1041. https://doi.org/10.1016/S0896-6273(03)00144-2

He, C., Peelen, M. V., Han, Z., Lin, N., Caramazza, A., \& Bi, Y. (2013). Selectivity for large nonmanipulable objects in scene-selective visual cortex does not require visual experience. NeuroImage, 79, 1-9. https://doi.org/10.1016/j.neuroimage.2013.04.051

Herbet, G., Moritz-Gasser, S., Boiseau, M., Duvaux, S., Cochereau, J., \& Duffau, H. (2016). Converging evidence for a cortico-subcortical network mediating lexical retrieval. Brain, 139(11), 3007-3021. https://doi.org/10.1093/brain/aww220

Hutchison, R. M., Culham, J. C., Everling, S., Flanagan, J. R., \& Gallivan, J. P. (2014). Distinct and distributed functional connectivity patterns across cortex reflect the domain-specific constraints of object, face, scene, body, and tool category-selective modules in the ventral visual pathway. NeuroImage, 96, 216-236. https://doi.org/10.1016/j.neuroimage.2014.03.068

Ishai, A., Ungerleider, L. G., Martin, A., Schouten, J. L., \& Haxby, J. V. (1999). Distributed representation of objects in the human ventral visual pathway. Proceedings of the National Academy of Sciences, 96(16), 9379-9384. https://doi.org/10.1073/pnas.96.16.9379

James, K. H., James, T. W., Jobard, G., Wong, A. C. N., \& Gauthier, I. (2005). Letter processing in the visual system: different activation patterns for single letters and strings. Cognitive, Affective \& Behavioral Neuroscience, 5(4), 452-466.

Jastorff, J., \& Orban, G. A. (2009). Human Functional Magnetic Resonance Imaging Reveals Separation and Integration of Shape and Motion Cues in Biological Motion Processing. Journal of Neuroscience, 29(22), 7315-7329. https://doi.org/10.1523/JNEUROSCI.4870-08.2009

Kanwisher, N., McDermott, J., \& Chun, M. M. (1997). The Fusiform Face Area: A Module in Human Extrastriate Cortex Specialized for Face Perception. The Journal of Neuroscience, 17(11), 4302-4311.

Kolster, H., Mandeville, J. B., Arsenault, J. T., Ekstrom, L. B., Wald, L. L., \& Vanduffel, W. (2009). Visual Field Map Clusters in Macaque Extrastriate Visual Cortex. Journal of Neuroscience, 29(21), 7031-7039. https://doi.org/10.1523/JNEUROSCI.0518-09.2009

Kolster, H., Peeters, R., \& Orban, G. A. (2010). The retinotopic organization of the human middle temporal area MT/V5 and its cortical neighbors. The Journal of Neuroscience: The Official Journal of the Society for Neuroscience, 30(29), 9801-9820. https://doi.org/10.1523/JNEUROSCI.2069-10.2010

Konkle, T., \& Caramazza, A. (2013). Tripartite Organization of the Ventral Stream by Animacy and Object Size. Journal of Neuroscience, 33(25), 10235-10242. https://doi.org/10.1523/JNEUROSCI.0983-13.2013

Konkle, T., \& Oliva, A. (2012). A Real-World Size Organization of Object Responses in Occipitotemporal Cortex. Neuron, 74(6), 1114-1124. https://doi.org/10.1016/j.neuron.2012.04.036

Kourtzi, Z., Bülthoff, H. H., Erb, M., \& Grodd, W. (2002). Object-selective responses in the human motion area MT/MST. Nature Neuroscience, 5(1), 17-18. https://doi.org/10.1038/nn780 
Lafer-Sousa, R., Conway, B. R., \& Kanwisher, N. G. (2016). Color-Biased Regions of the Ventral Visual Pathway Lie between Face- and Place-Selective Regions in Humans, as in Macaques. Journal of Neuroscience, 36(5), 1682-1697. https://doi.org/10.1523/JNEUROSCI.316415.2016

Liebenthal, E., Binder, J. R., Spitzer, S. M., Possing, E. T., \& Medler, D. A. (2005). Neural Substrates of Phonemic Perception. Cerebral Cortex, 15(10), 1621-1631. https://doi.org/10.1093/cercor/bhi040

Lingnau, A., \& Downing, P. E. (2015). The lateral occipitotemporal cortex in action. Trends in Cognitive Sciences, 19(5), 268-277. https://doi.org/10.1016/j.tics.2015.03.006

Lochy, A., Jacques, C., Maillard, L., Colnat-Coulbois, S., Rossion, B., \& Jonas, J. (2018). Selective visual representation of letters and words in the left ventral occipito-temporal cortex with intracerebral recordings. Proceedings of the National Academy of Sciences of the United States of America. https://doi.org/10.1073/pnas.1718987115

Marinus, E., \& de Jong, P. F. (2010). Variability in the word-reading performance of dyslexic readers: effects of letter length, phoneme length and digraph presence. Cortex; a Journal Devoted to the Study of the Nervous System and Behavior, 46(10), 1259-1271.

https://doi.org/10.1016/j.cortex.2010.06.005

Nordt, M., Gomez, J., Natu, V., Jeska, B., Barnett, M., \& Grill-Spector, K. (2018). Learning to Read Increases the Informativeness of Distributed Ventral Temporal Responses. Cerebral Cortex (New York, N.Y.: 1991). https://doi.org/10.1093/cercor/bhy178

Oishi, K., Huang, H., Yoshioka, T., Ying, S. H., Zee, D. S., Zilles, K., ... Mori, S. (2011). Superficially Located White Matter Structures Commonly Seen in the Human and the Macaque Brain with Diffusion Tensor Imaging. Brain Connectivity, 1(1), 37-47. https://doi.org/10.1089/brain.2011.0005

Orlov, T., Makin, T. R., \& Zohary, E. (2010). Topographic representation of the human body in the occipitotemporal cortex. Neuron, 68(3), 586-600. https://doi.org/10.1016/j.neuron.2010.09.032

Peelen, M. V., \& Downing, P. E. (2005). Selectivity for the Human Body in the Fusiform Gyrus. Journal of Neurophysiology, 93(1), 603-608. https://doi.org/10.1152/jn.00513.2004

Pietrini, P., Furey, M. L., Ricciardi, E., Gobbini, M. I., Wu, W.-H. C., Cohen, L., ... Haxby, J. V. (2004). Beyond sensory images: Object-based representation in the human ventral pathway. Proceedings of the National Academy of Sciences, 101(15), 5658-5663. https://doi.org/10.1073/ pnas.0400707101

Price, C. J., \& Devlin, J. T. (2011). The Interactive Account of ventral occipitotemporal contributions to reading. Trends in Cognitive Sciences, 15(6), 246-253.

https://doi.org/10.1016/j.tics.2011.04.001

Pugh, K. R., Landi, N., Preston, J. L., Mencl, W. E., Austin, A. C., Sibley, D., ... Frost, S. J. (2013). The relationship between phonological and auditory processing and brain organization in beginning readers. Brain and Language, 125(2), 173-183.

https://doi.org/10.1016/j.bandl.2012.04.004

Pyles, J. A., Garcia, J. O., Hoffman, D. D., \& Grossman, E. D. (2007). Visual perception and neural correlates of novel 'biological motion.’ Vision Research, 47(21), 2786-2797. https://doi.org/10.1016/j.visres.2007.07.017 
Ross, P. D. (2014). Body form and body motion processing are dissociable in the visual pathways. Frontiers in Psychology, 5. https://doi.org/10.3389/fpsyg.2014.00767

Ross, P. D., de Gelder, B., Crabbe, F., \& Grosbras, M.-H. (2014). Body-selective areas in the visual cortex are less active in children than in adults. Frontiers in Human Neuroscience, 8. https://doi.org/10.3389/fnhum.2014.00941

Rossion, B., Caldara, R., Seghier, M., Schuller, A.-M., Lazeyras, F., \& Mayer, E. (2003). A network of occipito-temporal face-sensitive areas besides the right middle fusiform gyrus is necessary for normal face processing. Brain, 126(11), 2381-2395. https://doi.org/10.1093/brain/awg241

Safford, A. S., Hussey, E. A., Parasuraman, R., \& Thompson, J. C. (2010). Object-Based Attentional Modulation of Biological Motion Processing: Spatiotemporal Dynamics Using Functional Magnetic Resonance Imaging and Electroencephalography. Journal of Neuroscience, 30(27), 9064-9073. https://doi.org/10.1523/JNEUROSCI.1779-10.2010

Saygin, Z. M., Osher, D. E., Norton, E. S., Youssoufian, D. A., Beach, S. D., Feather, J., ... Kanwisher, N. (2016). Connectivity precedes function in the development of the visual word form area. Nature Neuroscience, 19(9), 1250-1255. https://doi.org/10.1038/nn.4354

Schlaggar, B. L., \& McCandliss, B. D. (2007). Development of Neural Systems for Reading. Annual Review of Neuroscience, 30(1), 475-503. https://doi.org/10.1146/annurev.neuro.28.061604.135645

Sebastian, R., Gomez, Y., Leigh, R., Davis, C., Newhart, M., \& Hillis, A. E. (2014). The Roles of Occipitotemporal Cortex in Reading, Spelling, and Naming. Cognitive Neuropsychology, 31(0), 511-528. https://doi.org/10.1080/02643294.2014.884060

Silson, E. H., McKeefry, D. J., Rodgers, J., Gouws, A. D., Hymers, M., \& Morland, A. B. (2013). Specialized and independent processing of orientation and shape in visual field maps LO1 and LO2. Nature Neuroscience, 16(3), 267-269. https://doi.org/10.1038/nn.3327

Singer, J. M., Madsen, J. R., Anderson, W. S., \& Kreiman, G. (2015). Sensitivity to timing and order in human visual cortex. Journal of Neurophysiology, 113(5), 1656-1669. https://doi.org/10.1152/jn.00556.2014

Stevens, W. D., Kravitz, D. J., Peng, C. S., Tessler, M. H., \& Martin, A. (2017). Privileged Functional Connectivity between the Visual Word Form Area and the Language System. The Journal of Neuroscience, 37(21), 5288-5297. https://doi.org/10.1523/JNEUROSCI.0138-17.2017

Stevens, W. D., Tessler, M. H., Peng, C. S., \& Martin, A. (2015). Functional connectivity constrains the category-related organization of human ventral occipitotemporal cortex. Human Brain Mapping, 36(6), 2187-2206. https://doi.org/10.1002/hbm.22764

Strother, L., Coros, A. M., \& Vilis, T. (2016). Visual Cortical Representation of Whole Words and Hemifield-split Word Parts. Journal of Cognitive Neuroscience, 28(2), 252-260. https://doi.org/ 10.1162/jocn_a_00900

Strother, L., Zhou, Z., Coros, A. K., \& Vilis, T. (2017). An fMRI study of visual hemifield integration and cerebral lateralization. Neuropsychologia, 100, 35-43. https://doi.org/10.1016/j.neuropsychologia.2017.04.003

Tootell, R. B., Echavarria, C., \& Nasr, S. (2015). A problem of overlap. Visual Neuroscience, 32. https://doi.org/10.1017/S0952523814000340 
Vangeneugden, J., Peelen, M. V., Tadin, D., \& Battelli, L. (2014). Distinct neural mechanisms for body form and body motion discriminations. The Journal of Neuroscience: The Official Journal of the Society for Neuroscience, 34(2), 574-585. https://doi.org/10.1523/JNEUROSCI.403213.2014

Vinckier, F., Dehaene, S., Jobert, A., Dubus, J. P., Sigman, M., \& Cohen, L. (2007). Hierarchical coding of letter strings in the ventral stream: dissecting the inner organization of the visual word-form system. Neuron, 55(1), 143-156. https://doi.org/10.1016/j.neuron.2007.05.031

Vogel, A. C., Petersen, S. E., \& Schlaggar, B. L. (2014). The VWFA: it's not just for words anymore. Frontiers in Human Neuroscience, 8. https://doi.org/10.3389/fnhum.2014.00088

Vuilleumier, P., Henson, R. N., Driver, J., \& Dolan, R. J. (2002). Multiple levels of visual object constancy revealed by event-related fMRI of repetition priming. Nature Neuroscience, 5(5), 491-499. https://doi.org/10.1038/nn839

Weiner, K. S., Barnett, M. A., Lorenz, S., Caspers, J., Stigliani, A., Amunts, K., ... Grill-Spector, K. (2017). The Cytoarchitecture of Domain-specific Regions in Human High-level Visual Cortex. Cerebral Cortex, 27(1), 146-161. https://doi.org/10.1093/cercor/bhw361

Weiner, K. S., \& Grill-Spector, K. (2010). Sparsely-distributed organization of face and limb activations in human ventral temporal cortex. NeuroImage, 52(4), 1559-1573. https://doi.org/10.1016/j.neuroimage.2010.04.262

Weiner, K. S., \& Grill-Spector, K. (2013). Neural representations of faces and limbs neighbor in human high-level visual cortex: evidence for a new organization principle. Psychological Research, 77(1), 74-97. https://doi.org/10.1007/s00426-011-0392-x

Wheatley, T., Weisberg, J., Beauchamp, M. S., \& Martin, A. (2005). Automatic Priming of Semantically Related Words Reduces Activity in the Fusiform Gyrus. Journal of Cognitive Neuroscience, 17(12), 1871-1885. https://doi.org/10.1162/089892905775008689

Whitney, C. (2001). How the brain encodes the order of letters in a printed word: the SERIOL model and selective literature review. Psychonomic Bulletin \& Review, 8(2), 221-243.

Whitney, C. (2017). When serial letter processing implies a facilitative length effect. Language, Cognition and Neuroscience, 0(0), 1-6. https://doi.org/10.1080/23273798.2017.1404115

Whitney, C., \& Marton, Y. (2013). The SERIOL2 model of orthographic processing. Retrieved from http://files.eric.ed.gov/fulltext/ED543279.pdf

Wiggett, A. J., Pritchard, I. C., \& Downing, P. E. (2009). Animate and inanimate objects in human visual cortex: Evidence for task-independent category effects. Neuropsychologia, 47(14), 31113117. https://doi.org/10.1016/j.neuropsychologia.2009.07.008

Yeatman, J. D., Rauschecker, A. M., \& Wandell, B. A. (2013). Anatomy of the visual word form area: Adjacent cortical circuits and long-range white matter connections. Brain and Language, 125(2), 146-155. https://doi.org/10.1016/j.bandl.2012.04.010

Zoccolotti, P., De Luca, M., Di Pace, E., Gasperini, F., Judica, A., \& Spinelli, D. (2005). Word length effect in early reading and in developmental dyslexia. Brain and Language, 93(3), 369-373. https://doi.org/10.1016/j.bandl.2004.10.010 